\title{
IdeAs
}

Idées d'Amériques

$6 \mid 2015$

Migrer dans les Amériques

\section{Cathal Doyle et Jill Cariño, Les Peuples autochtones et les industries extractives. Mettre en œuvre le Consentement, libre, préalable et informé} Paris, l'Harmattan, 2014, 186 p.

\section{Sandrine Tolazzi}

\section{OpenEdition}

\section{Journals}

Édition électronique

URL : https://journals.openedition.org/ideas/1247

DOI : $10.4000 /$ ideas. 1247

ISSN : $1950-5701$

Éditeur

Institut des Amériques

Référence électronique

Sandrine Tolazzi, « Cathal Doyle et Jill Cariño, Les Peuples autochtones et les industries extractives. Mettre en œuvre le Consentement, libre, préalable et informé », IdeAs [En ligne], 6 | 2015, mis en ligne le 17 décembre 2015, consulté le 19 octobre 2022. URL : http://journals.openedition.org/ideas/1247 ; DOI : https://doi.org/10.4000/ideas.1247

Ce document a été généré automatiquement le 19 octobre 2022

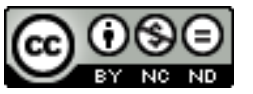

Creative Commons - Attribution - Pas d'Utilisation Commerciale - Pas de Modification 4.0 International - CC BY-NC-ND 4.0

https://creativecommons.org/licenses/by-nc-nd/4.0/ 


\section{Cathal Doyle et Jill Cariño, Les Peuples autochtones et les industries extractives. Mettre en œuvre le Consentement, libre, préalable et informé}

Paris, l'Harmattan, 2014, 186 p.

Sandrine Tolazzi

\section{RÉFÉRENCE}

Cathal Doyle et Jill Cariño, Les Peuples autochtones et les industries extractives. Mettre en œuvre le Consentement, libre, préalable et informé, Paris, l'Harmattan, 2014, 186 p.

Sous l'action conjuguée d'une forte demande en matières premières et de l'augmentation du prix de l'énergie et des produits de base de l'industrie, le secteur extractif connaît une croissance exponentielle depuis une dizaine d'années environ, et ce sur tous les continents. Les nouvelles technologies d'extraction rendent possible l'exploitation de gisements à très faible rendement, et conduisent à une multiplication du nombre de mines à ciel ouvert sur des surfaces de plus en plus importantes. Outre le spectre de l'épuisement des ressources non renouvelables ou de l' "après-boom minier", l'essor de l'industrie extractive soulève la question de son impact environnemental : paysages irrémédiablement altérés, écosystèmes perturbés, eau et air contaminés, augmentation des émissions de gaz à effet de serre... autant de conséquences qui touchent de manière encore plus directe les populations vivant à proximité des projets miniers. Or, il se trouve qu'un grand nombre de ces derniers se situent en des endroits occupés par des autochtones, et que les communautés qui y vivent souhaitent pouvoir exercer leur souveraineté lorsqu'il est question de 
développement sur ce qu'elles considèrent comme leur territoire et leurs ressources. Dans ce contexte, les auteurs de cet ouvrage - Cathal Doyle et Jill Cariño - se sont penchés sur la relation entre entreprises minières et populations autochtones dans le cadre des nouveaux projets miniers afin d'étudier la manière de mettre en œuvre le consentement libre, préalable et informé de ces populations à ces projets.

2 Selon les auteurs, le consentement libre, préalable et informé (ou FPIC - free, prior, and informed consent) découle directement des droits à l'autodétermination qui figurent parmi les normes internationales, et se retrouvent en particulier dans la Déclaration des Nations unies sur les peuples autochtones. Cette insistance sur le cadre juridique international se retrouve dans l'ensemble de l'ouvrage, et en particulier dans la première partie qui vise à démontrer la manière dont l'exigence du consentement des peuples autochtones, bien qu'elle n'y figure pas explicitement, provient naturellement des droits tels que formulés dans les Conventions internationales. Le respect de ce cadre juridique international voudrait donc que les entreprises aient systématiquement recours au FPIC dans le contexte de nouveaux projets miniers, y compris lorsque le gouvernement refuse lui-même d'appliquer ces normes. Partant de ce principe selon lequel les entreprises doivent agir selon les standards internationaux, le rapport souhaite fournir des pistes quant à la mise en œuvre du FPIC en se basant sur le point de vue des autochtones mais aussi sur celui des entreprises.

Doyle et Cariño notent ainsi que pour les populations autochtones, le droit à être consulté proviendrait avant tout de la pratique coutumière de demander l'autorisation avant de réaliser une activité. La mise en œuvre de ce droit implique le respect d'un certain nombre de principes, tels que la définition du processus par les populations concernées, l'implication des autorités traditionnelles de la communauté, l'inclusion de toutes les communautés affectées, l'accès à un conseil juridique ou technique indépendant pour pouvoir interpréter correctement les informations, ou encore la participation à l'évaluation des impacts environnementaux souvent réalisée par les entreprises. L'énoncé de ces principes ne manquera pas de faire s'interroger le lecteur sur les difficultés des entreprises à les appliquer : la nécessité de respecter le rythme des communautés en n'imposant aucune limite temporelle au processus de consultation, par exemple, est peu compatible avec le besoin pour les entreprises de respecter certaines contraintes de temps. D'autres points, comme le besoin de rechercher le FPIC le plus tôt possible et à toutes les étapes du projet, peuvent au final poser problème aux communautés elles-mêmes en conduisant, comme le notent les auteurs, à une "fatigue de consultation". Ainsi, la manière dont les populations autochtones envisagent le FPIC soulève un certain nombre de questionnements qui apparaissent au travers des différentes études de cas citées.

Quant au point de vue des entreprises tel qu'exprimé au cours des différents entretiens menés par les auteurs, il reflète d'emblée leur position ambiguë. Si elles cherchent en effet de plus en plus à obtenir le consentement des populations autochtones dans le cadre de leurs projets, leurs politiques ne garantissent absolument pas le FPIC, et les études de cas laissent à penser que les entreprises ont recours au FPIC lorsque les gouvernements le requièrent, lorsqu'elles sont déstabilisées par un conflit majeur qui les opposent à des populations autochtones soutenues par des acteurs de la société civile, ou lorsqu'elles cherchent à améliorer leur image de marque. De manière concrète, les entreprises font mention d'un certain nombre de difficultés (comment définir qui est autochtone? A quel moment exiger le consentement? Comment le FPIC 
s'articule-t-il avec la souveraineté nationale ?) et semblent incertaines sur l'attitude à adopter lorsque le FPIC n'est pas lui-même incorporé dans une législation nationale.

Cette dernière remarque fait apparaître un point crucial qui figure en toile de fond de l'ensemble de l'ouvrage sans être vraiment abordé en tant que tel. En effet, en se focalisant sur la relation entre entreprises et populations autochtones et en ancrant la légitimité du FPIC dans des normes internationales, les auteurs font l'impasse sur le rôle de l'État comme acteur fondamental dans les négociations autour des projets miniers. Pourtant, ils reconnaissent que pour les populations autochtones, «[le] plus grand obstacle au FPIC est le manque de reconnaissance par les États de la souveraineté des peuples autochtones sur leurs terres et sur leurs ressources » (Doyle C. \& Cariño J., $2014: 60)$ et soulignent le commentaire d'une entreprise affirmant que «les plus grandes difficultés sont peut-être l'absence d'une bonne gouvernance et de comportements clairs des autorités de l'État " (Doyle C. \& Cariño J., 2014 : 101). Mais leur solution à ce problème - encourager les entreprises à faire pression sur les États pour faire respecter le FPIC - semble utopique dans la mesure où, historiquement, les entreprises ont plutôt agi à l'inverse, poussant les États à adopter des législations restrictives par rapport aux droits territoriaux et à la souveraineté autochtones.

Ainsi, on peut se demander si le cadre international est à lui seul en mesure d'inciter les entreprises à mettre en œuvre le FPIC, en particulier lorsqu'il y a un risque d'opposition au projet envisagé de la part des communautés concernées. L'importance croissante donnée au consentement des populations autochtones par les entreprises mais aussi par le secteur financier représente certes un pas en avant, mais le manque d'exemples de réussite dans les cas étudiés peut néanmoins inciter au pessimisme. Les auteurs formulent toutefois des recommandations, y compris aux États ou aux organisations de la société civile pouvant également être partie prenante des projets de l'industrie extractive, qui permettent de répondre à leur objectif premier en fournissant une base de dialogue entre l'industrie et les populations autochtones concernant le FPIC. Cet objectif de dialogue permet au final d'expliquer pourquoi la critique de l'industrie extractive et des acteurs de cette industrie reste, dans cet ouvrage, plutôt timide, alors que leur mode de relation avec les populations autochtones peut encore être qualifiée de déplorable dans la grande majorité des cas.

\section{AUTEURS}

\section{SANDRINE TOLAZZI}

Sandrine Tolazzi est Maître de Conférences à l'UFR des Langues de l'Université Grenoble Alpes, où elle enseigne et dirige des mémoires de recherche sur les enjeux contemporains auxquels font face les sociétés canadienne et australienne. Elle est membre de l'unité de recherches ILCEA4 (Institut des Langues et des Cultures d'Europe, Amérique, Afrique, Asie et Australie) et dirige le Centre interdisciplinaire d'Études Canadiennes de Grenoble. Elle est également membre du Conseil d'Administration de l'Association Française d'Études Canadiennes (AFEC). Ses recherches portent actuellement sur le développement des ressources minières au Canada et en Australie, et 
plus particulièrement sur la façon dont ce développement vient redéfinir les rapports entre populations autochtones, acteurs gouvernementaux et entreprises ainsi que sur les questions d'appropriation du territoire dans les villes minières. 